\title{
Safety and Immunogenicity of a Recombinant Adenovirus Serotype 35-Vectored HIV-1 Vaccine in Adenovirus Serotype 5 Seronegative and Seropositive Individuals
}

\author{
Jonathan D Fuchs ${ }^{1,2, *}$, Pierre-Alexandre Bart ${ }^{3}$, Nicole Frahm ${ }^{4}$, Cecilia Morgan ${ }^{4}$, Peter B Gilbert ${ }^{4}$, Nidhi Kochar ${ }^{4}$, Stephen C DeRosa ${ }^{4}$, Georgia \\ D Tomaras ${ }^{5}$, Theresa M Wagner ${ }^{1}$, Lindsey R Baden ${ }^{6}$, Beryl A Koblin ${ }^{7}$, Nadine G Rouphael ${ }^{8}$, Spyros A Kalams ${ }^{9}$, Michael C Keefer ${ }^{10}$, Paul A \\ Goepfert $^{11}$, Magdalena E Sobieszczyk ${ }^{12}$, Kenneth H Mayer ${ }^{13}$, Edith Swann ${ }^{14}$, Hua-Xin Liao ${ }^{5}$, Barton F Haynes ${ }^{5}$, Barney S Graham ${ }^{15}$ and M $^{-}$ \\ Juliana McElrath ${ }^{4}$ for the NIAID HIV Vaccine Trials Network \\ ${ }^{1}$ Population Health Division, San Francisco Department of Public Health, San Francisco, CA, USA \\ ${ }^{2}$ Department of Medicine, University of California, San Francisco, San Francisco, USA \\ ${ }^{3}$ Centre Hospitalier Universitaire Vaudois, Lausanne, Switzerland \\ ${ }^{4}$ Vaccine and Infectious Disease Division, Fred Hutchinson Cancer Research Center, Seattle, WA, USA \\ ${ }^{5}$ Human Vaccine Institute, Duke University, Durham, NC, USA \\ ${ }^{6}$ Division of Infectious Disease, Brigham and Women's Hospital, Boston, MA, USA \\ ${ }^{7}$ Laboratory of Infectious Disease Prevention, New York Blood Center, New York, NY, USA \\ ${ }^{8}$ The Hope Clinic, Division of Infectious Diseases, Emory University, Atlanta, GA, USA \\ ${ }^{9}$ Infectious Diseases Division, Vanderbilt University School of Medicine, Nashville, TN, USA \\ ${ }^{10}$ University of Rochester School of Medicine and Dentistry, Rochester, NY, USA \\ ${ }^{11}$ Department of Medicine, University of Alabama at Birmingham, Birmingham, AL, USA \\ ${ }^{12}$ Division of Infectious Diseases, Columbia University College of Physicians and Surgeons, New York, NY, USA \\ ${ }^{13}$ Fenway Health and the Division of Infectious Diseases, Department of Medicine, Beth Israel Deaconess Medical Center, Harvard Medical School, USA \\ ${ }^{14}$ Division of AIDS, National Institute of Allergy and Infectious Diseases, Bethesda, MD, USA \\ ${ }^{15}$ Vaccine Research Center, National Institute of Allergy and Infectious Diseases, Bethesda, MD, USA
}

\begin{abstract}
Background: Recombinant adenovirus serotype 5 (rAd5)-vectored HIV-1 vaccines have not prevented HIV-1 infection or disease and pre-existing Ad5 neutralizing antibodies may limit the clinical utility of Ad5 vectors globally. Using a rare Ad serotype vector, such as Ad35, may circumvent these issues, but there are few data on the safety and immunogenicity of rAd35 directly compared to rAd5 following human vaccination.

Methods: HVTN 077 randomized 192 healthy, HIV-uninfected participants into one of four HIV-1 vaccine/placebo groups: rAd35/rAd5, DNA/rAd5, and DNA/rAd35 in Ad5-seronegative persons; and DNA/rAd35 in Ad5-seropositive persons. All vaccines encoded the HIV-1 EnvA antigen. Antibody and T-cell responses were measured 4 weeks post boost immunization.

Results: All vaccines were generally well tolerated and similarly immunogenic. As compared to rAd5, rAd35 was equally potent in boosting HIV-1-specific humoral and cellular immunity and responses were not significantly attenuated in those with baseline Ad5 seropositivity. Like DNA, rAd35 efficiently primed rAd5 boosting. All vaccine regimens tested elicited cross-clade antibody responses, including Env V1/V2-specific IgG responses.

Conclusions: Vaccine antigen delivery by rAd35 is well-tolerated and immunogenic as a prime to rAd5 immunization and as a boost to prior DNA immunization with the homologous insert. Further development of rAd35vectored prime-boost vaccine regimens is warranted.
\end{abstract}

Keywords: HIV Vaccine; Adenovirus 35; Adenovirus 5; DNA vaccine; Randomized clinical trial

\section{Introduction}

The development of a safe and effective preventive HIV vaccine remains an urgent public health priority in the setting of an estimated 2.1 million new infections globally [1]. Since the first preventive HIV vaccine candidate entered clinical testing in 1987, four distinct vaccine concepts including subunit protein, DNA, and viral vector vaccines have been evaluated in six completed efficacy trials [2-7]. Thus far, only one vaccine regimen, a canarypox vector encoding three HIV-1 genes with a gp120 subunit boost, demonstrated partial efficacy in a phase $2 \mathrm{~b}$ study [6]. Subsequent findings indicated that non-neutralizing IgG antibody responses to HIV-1 Env V1/V2 were significantly correlated with decreased risk of infection in vaccines [8]

HIV vaccine candidates using recombinant adenovirus (rAd) vectors have been some of the most immunogenic [9]. The Step study (HIV Vaccine Trials Network [HVTN] 502) was the first efficacy trial to test an rAd5-vectored vaccine expressing HIV-1 clade B Gag, Pol and Nef; however the product failed to protect against infection or disease progression. Furthermore, this study suggested that pre-existing Ad5 neutralizing antibodies may have played a role in increased HIV susceptibility among vaccinees $[4,10]$. A multiclade/multigene DNA prime, rAd5 boost regimen encoding HIV-1 Gag, Pol, and Nef from

*Corresponding author: Jonathan D Fuchs, San Francisco Department of Public Health, 25 Van Ness Ave, Suite 100, San Francisco, CA, 94102, USA, Tel: 01415 336-1290; E-mail: jonathan.fuchs@sfdph.org

Received April 09, 2015; Accepted May 12, 2015; Published May 23, 2015

Citation: Fuchs JD, Bart PA, Frahm N, Morgan C, Gilbert PB, et al. (2015) Safety and Immunogenicity of a Recombinant Adenovirus Serotype 35-Vectored HIV-1 Vaccine in Adenovirus Serotype 5 Seronegative and Seropositive Individuals. J AIDS Clin Res 6: 461. doi:10.4172/2155-6113.1000461

Copyright: (C) 2015 Fuchs JD, et al. This is an open-access article distributed under the terms of the Creative Commons Attribution License, which permits unrestricted use, distribution, and reproduction in any medium, provided the original author and source are credited. 
Citation: Fuchs JD, Bart PA, Frahm N, Morgan C, Gilbert PB, et al. (2015) Safety and Immunogenicity of a Recombinant Adenovirus Serotype 35-Vectored HIV-1 Vaccine in Adenovirus Serotype 5 Seronegative and Seropositive Individuals. J AIDS Clin Res 6: 461. doi:10.4172/21556113.1000461

Page 2 of 10

clade B, and Env from clades A, B, and C developed by the NIAID Vaccine Research Center (VRC) was immunogenic in early phase trials $[11,12]$. However, the recent HVTN 505 phase 2b trial (NCT00865566) evaluating this regimen failed to show reduced HIV acquisition or viral load setpoint in Ad5-seronegative, circumcised men who have sex with men and transgender women from the United States [7]. Even if such a vaccine were shown effective in this targeted population, preexisting neutralizing antibodies to Ad5 globally are highly prevalent, particularly in the developing world [13-16]. Thus, an Ad5-based HIV vaccine would have limited clinical utility in many of the settings where an HIV vaccine is needed the most.

While replication-defective rAd5-based products are unlikely to move forward as candidate HIV vaccines, substantial interest remains in evaluating potentially less common adenoviral serotype vectors, such as $\operatorname{Ad} 26$ and Ad35. For example, compared to Ad5, Ad35 has a relatively low global seroprevalence $[13,14,16]$ and unique tropism [17] that offer the potential to circumvent pre-existing immunity and confer protection against infection as seen in non-human primate studies [18]. Consequently, Ad35 serves as the viral vector for several vaccine candidates to prevent HIV [19], tuberculosis [20], and hepatitis C virus infections [21].

The VRC developed an experimental HIV-1 Env clade A-expressing recombinant Ad35 (rAd35) vaccine which was well tolerated and immunogenic in a phase I trial [22]. Compared to three DNA immunizations, a single rAd35 priming immunization would reduce the number of injections required, supporting greater acceptability and uptake if shown effective and licensed for use. Animal models suggest that Ad35 is not cross-neutralized by antibodies to Ad5 [15,23] and that Ad35-vectored vaccines retain their immunogenicity in the setting of pre-existing Ad5 neutralizing antibodies [23]. However, it is unknown whether these observations extend to humans. The HVTN conducted a phase Ib study to further evaluate the safety of the VRC prototype rAd35-vectored HIV vaccine, and to 1) compare the HIVspecific immune responses elicited by a heterologous vector regimen (rAd35 prime/rAd5 boost) versus a DNA prime, rAd5 boost regimen; 2) to determine whether rAd35 is as potent as rAd5 as a boost following DNA priming; and 3) to assess whether pre-existing Ad5 neutralizing antibodies affect the HIV-specific immune responses to the rAd35delivered EnvA antigen.

\section{Subjects, Materials and Methods}

\section{Vaccines}

All vaccines tested in HVTN 077 were produced by the NIH VRC and GenVec, Inc. (Gaithersburg, Maryland). One prototype vaccine consisted of a single E1-deleted, replication-deficient group B rAd35 vector, constructed to express a truncated modified HIV-1 clade A Env. The full E1, E3 and partial E4 deleted replication-deficient recombinant group $\mathrm{C}$ Ad5 vector also expressed the same truncated modified version of the HIV-1 clade A env gene. Both vaccines were formulated at a dose of $1 \times 10^{10}$ particle units and administered by needle and syringe intramuscularly.

The DNA-EnvA vaccine encodes for the clade A env gene and is one of the 6 plasmids included in HVTN 505 regimen [7]. The DNA vaccination was administered intramuscularly via the needle free injection device Biojector 2000 (Tualitin, Oregon) at a dose of $4 \mathrm{mg}$. The placebos for the adenovectors and DNA vaccines were final formulation buffer and phosphate-buffered saline (PBS), respectively.

\section{Study design and procedures}

HVTN 077 was a randomized, double-blind, placebo-controlled

\begin{tabular}{|c|c|c|c|c|c|c|}
\hline \multirow{2}{*}{$\begin{array}{c}\text { Treatment } \\
\text { Group }\end{array}$} & \multirow{2}{*}{ Ad5 nAb* } & \multirow{2}{*}{$\mathbf{N}^{\star *}$} & \multicolumn{4}{|c|}{ Injection schedule months (days) } \\
\cline { 4 - 7 } & & $\mathbf{0}(\mathbf{0})$ & $\mathbf{1}(\mathbf{2 8})$ & $\mathbf{2} \mathbf{( 5 6 )}$ & $\mathbf{6}(\mathbf{1 6 8})$ \\
\hline 1 & $<18$ & $34 / 6$ & rAd5 & - & - & rAd5 \\
\hline 2 & $<18$ & $48 / 8$ & DNA & DNA & DNA & rAd5 \\
\hline 3 & $<18$ & $48 / 8$ & DNA & DNA & DNA & rAd35 \\
\hline 4 & $\geq 18$ & $34 / 6$ & DNA & DNA & DNA & rAd35 \\
\hline Total & & 192 & & & & \\
\hline
\end{tabular}

*Adenovirus 5 (Ad5) neutralizing antibody $(\mathrm{nAb}) \geq 18$ represents $\mathrm{Ad} 5$ seropositive individuals; ${ }^{* *} \mathrm{~N}$ represents the active vaccinees/placebo recipients who were blinded to treatment assignment within each treatment group. DNA vaccinations were delivered by Biojector, and adenovectors were delivered by needle and syringe. Groups 2 and 3 were blinded to assignment to these groups.

Table 1: HVTN 077 Protocol Schema.

phase $1 \mathrm{~b}$ trial conducted at 11 clinical sites in the United States. The protocol was approved by the institutional review boards of all participating centers (Clinical Trials.gov registration NCT00801697). Between February of 2009 and January 2010, 192 adults aged 18-50 who reported low risk for infection and determined to be HIV-1seronegative and healthy based on medical history, physical exam, and laboratory tests were enrolled after providing written informed consent. Eligible individuals who consented and enrolled were randomized to one of four treatment $(\mathrm{T})$ groups (Table 1). Individuals randomized to treatment groups 2 (DNA/rAd5) or 3 (DNA/rAd35) were blinded to their assignment. For all groups, participants were blinded to assignment to vaccine or placebo. All participants were Ad35 neutralizing antibody $(\mathrm{nAb})$ negative at baseline; for groups 1-3, participants were also Ad5 nAb negative. In group 4, participants were Ad $5 \mathrm{nAb}$ positive determined by $\mathrm{nAb}$ titers $\geq 18$.

Safety evaluations included physical examinations and standard clinical chemistry and hematological tests. Local injection site (pain, tenderness, redness, erythema, and induration) and systemic (malaise, headache, fever, chills, myalgias, arthralgias, nausea, vomiting, and fatigue) reactogenicity symptoms were assessed for three days following each vaccination or until resolution. Adverse events were graded based on the HVTN Table for Grading Severity of Adverse Experiences (http://rsc.tech-res.com/Document/safetyandpharmacovigilance/ Table_For_Grading_Severity_of_Adult_Pediatric__Adverse_Events. pdf). Several licensed diagnostic HIV ELISA assays (Abbott HIVAB HIV 1/2 [rDNA], Abbott Architect HIV Ag/Ab Combo, BioRad Genetic System HIV 1/2 Plus O EIA, BioRad Genetic System HIV 1/2 rLAV, and BioRad Multispot HIV-1/HIV-2 Rapid Test) were performed on sera on all participants at the end of study (Day 364) to assess vaccine-induced seroreactivity.

Blood samples for assessment for primary immunogenicity were collected at days 28 ( 4 weeks after the single rAd35 priming injection in Group 1), 84 (4 weeks after the DNA priming series in Groups 2-4) and 196 (4 weeks after the boost vaccination in all groups).

\section{Immune response assays}

\section{Humoral responses}

Neutralizing Antibodies to Ad5 and Ad35: Baseline Ad5 neutralizing antibody titers were measured as previously described with titers $\geq 18$ noted as positive [24]. Ad35 neutralizing antibody titers were measured by luciferase transgene detection [25], and titers $\geq 12$ noted as positive.

HIV-Specific Binding Antibody Assays: Validated binding antibody multiplex assays [26] for measurement of vaccine elicited 
Citation: Fuchs JD, Bart PA, Frahm N, Morgan C, Gilbert PB, et al. (2015) Safety and Immunogenicity of a Recombinant Adenovirus Serotype 35-Vectored HIV-1 Vaccine in Adenovirus Serotype 5 Seronegative and Seropositive Individuals. J AIDS Clin Res 6: 461. doi:10.4172/21556113.1000461

Page 3 of 10

HIV-1 Envelope-specific IgG to Group M Consensus (Con S gp140 CFI), Clade A (00MSA 4076 gp140), Clade B (B.con.env03 $140 \mathrm{CF}$ ), and Clade C (C.con.env03 $140 \mathrm{CF}$ ) were performed according to a pre-specified assay study plan following GCLP guidelines. Additional studies were performed for Env V1V2 reactive antibodies [8] utilizing scaffolds gp70 V1V2 VRC EnvA [27] and gp70 V1V2 (Case A2) [28]. HIV-1-specific IgG was detected from 1:50 serum dilution with biotinconjugated mouse anti-human IgG (Southern Biotech, Birmingham, AL) $(4 \mu \mathrm{g} / \mathrm{ml})$, followed by washing and incubation with streptavidinPE (BD Pharmingen). Mean fluorescent intensity (MFI) readouts were acquired on a Bio-Plex instrument (BioRad). Positive controls (purified HIV-1 positive immunoglobulin [HIVIG] and CH58 mAb [27] for the V1V2 assays) and negative controls (blank beads, HIV-1 negative sample, and baseline samples) were included to ensure specificity and for maintaining consistency and reproducibility between assays.

Positivity of antibody binding at Day 196 was defined by meeting all three conditions: (1) the MFI minus blank values are $\geq$ antigen specific cutoff (based on the average +3 standard deviations of 80 seronegative plasma samples), (2) the MFI minus blank values are greater than 3 times the baseline (Day 0) MFI minus blank values, and (3) the MFI values are greater than three times the baseline MFI values. For positive responses, binding magnitude was quantified by the net MFI concentration (subtracting the blank value) estimated using a 10-point standard curve (4PL fit).

T Cell Response: Peripheral blood mononuclear cells (PBMC) were isolated and cryopreserved from whole blood within 8 hours of venipuncture using standard procedures [29]. A 10-color intracellular cytokine staining (ICS) assay was performed on cryopreserved PBMC as previously described [30-32]. For the detection of HIV-specific T cells, thawed PBMC were rested overnight and then stimulated for 6 hours with overlapping HIV-1 15-mer peptide pools matched to the vaccine insert (VRC EnvA). Positivity was established at $\mathrm{p}<10^{-5}$ using a Fisher's exact test comparing stimulated and unstimulated samples.

\section{Statistical analysis}

All data from enrolled participants who received at least one vaccination were analyzed. Five study groups were evaluated for immunogenicity: the four vaccinated groups individually plus the pooled placebo groups. HIV-1 specific IgG binding antibody and T-cell responses were evaluated at baseline (Day 0, IgG only) and at the primary immunogenicity timepoint, Day 196 (one month after the final injection). Rates of HIV-1 Env-specific antibodies and positive CD4+ and CD8+ T-cell responses (for cells expressing IFN- $\gamma$ and/or IL-2 as measured by ICS) were estimated for each study group and timepoint. Lachenbruch's test was used for comparing primary immunogenicity endpoints between study groups [33]. Response rates were compared between groups using Fisher's exact tests. Magnitudes of responses among positive responders were compared between study groups using Wilcoxon rank sum tests. All statistical tests were 2-sided. Primary and secondary analyses comparing immunogenicity endpoints between vaccinated groups were considered statistically significant if $p \leq 0.033$, chosen to control the overall type I error rate at 0.10 and correcting for three pairwise comparisons. All other analyses used $\mathrm{p} \leq 0.05$ to judge statistical significance. All descriptive and inferential statistical analyses were performed using SAS and/or R statistical software.

\section{Results}

\section{Participant accrual, demographic data, and vaccine safety}

Of the 736 individuals who underwent screening procedures,

\begin{tabular}{|c|c|c|c|c|c|}
\hline \multicolumn{3}{|c|}{ Ad5 } & \multicolumn{3}{|c|}{ Ad35 } \\
\hline Titer & N & Frequency & Titer & N & Frequency \\
\hline$<18$ & 431 & $59.7 \%$ & $<12$ & 670 & $91 \%$ \\
\hline $18-100$ & 40 & $5.5 \%$ & $12-100$ & 38 & $5.2 \%$ \\
\hline $101-1000$ & 156 & $21.6 \%$ & $101-1000$ & 14 & $1.9 \%$ \\
\hline $1001-4608$ & 80 & $11.1 \%$ & $1001-8748$ & 13 & $1.7 \%$ \\
\hline$>4608$ & 15 & $2.1 \%$ & $>8748$ & 1 & $0.20 \%$ \\
\hline Total $^{*}$ & 722 & $100 \%$ & Total & 736 & $100 \%$ \\
\hline
\end{tabular}

*Ad5 neutralizing antibody titers were unavailable for fourteen individuals who underwent Ad35 neutralizing antibody screening.

Table 2: Frequency and titers of neutralizing antibody to Ad5 and Ad35 among those screening for trial eligibility.

40.3\% had detectable neutralizing antibodies to Ad5 whereas only 9\% were Ad35 seropositive (Table 2). The median age of the 192 enrolled participants was 28 years; $42 \%$ were women, $65 \%$ were nonHispanic whites, $8 \%$ were Hispanic, and $16 \%$ Non-Hispanic Black. All participants received their initial vaccination and $98 \%$ received the second vaccination; of those assigned to 4 injections, $94 \%$ received the third, and $90 \%$ all four vaccinations. No significant differences were observed in vaccine completion rates between treatment groups. The primary reasons for study discontinuation included loss to follow-up or participant relocation $(n=9)$, incarceration $(n=1)$, refusal $(n=2)$ or other reasons $(n=2)$; none were due to adverse experiences or reactogenicity related to vaccination.

Overall, each of the vaccine components was well tolerated. Pain/and or tenderness at the injection site was reported most commonly ( $88 \%$ of study participants). Differences between groups were detected; as seen in Figure 1, those receiving the $\mathrm{rAd} 35 / \mathrm{rAd} 5$ heterologous adenovector regimen were less likely to report local reactions compared to the DNA prime, adenovector boost regimens $(\mathrm{p}<0.001)$. Maximum systemic symptoms were less commonly reported by those with pre-existing neutralizing antibodies to Ad5 (T4, Figure 1). There were 22 adverse events that were at least probably or definitely attributed to the vaccine, and most were local injection site reactions that were characterized as mild or moderate in severity. One case of transient mild leukopenia deemed probably related to vaccination was observed after receiving DNA in a T4 participant. Four expedited adverse events were reported in the trial including rectal bleeding, post traumatic lower extremity and right intraorbital ethmoid fractures, bipolar disorder, and gallstone pancreatitis; none were attributed to product. No significant differences in laboratory parameters were noted among groups. Overall, $60 \%$ had evidence of vaccine-induced HIV seroreactivity at the end of study using several commercially available HIV test kits. Rates were highest among $\mathrm{rAd} 35 / \mathrm{rAd} 5$ vaccinees $(81.8 \%)$ and lowest among participants who received DNA/rAd5 (45.7\%).

\section{HIV-1-specific antibody responses}

Each of the vaccine regimens induced high frequency and magnitude cross-clade binding antibody responses (Figure 2). The antibody response rates were $100 \%$ for all treatment groups recognizing the consensus M gp140 (data not shown) and 97-100\% recognizing the clade A Env antigen. For the clade B antigen, responses were also detected in greater than $92 \%$ of individuals. For the clade $\mathrm{C}$ antigen, response frequencies were highest for $\mathrm{rAd} 35 / \mathrm{rAd} 5$ (96\%) compared to the DNA prime/adenovector boost groups (76-78\% for groups 2-4), however at lower magnitude in comparison to the DNA/rAd5 group $(\mathrm{p}=0.02)$.

Based on evidence that IgG binding antibodies to V1/V2 were correlated with reduced risk of HIV infection in the RV144 vaccine 
Citation: Fuchs JD, Bart PA, Frahm N, Morgan C, Gilbert PB, et al. (2015) Safety and Immunogenicity of a Recombinant Adenovirus Serotype 35-Vectored HIV-1 Vaccine in Adenovirus Serotype 5 Seronegative and Seropositive Individuals. J AIDS Clin Res 6: 461. doi:10.4172/21556113.1000461

A
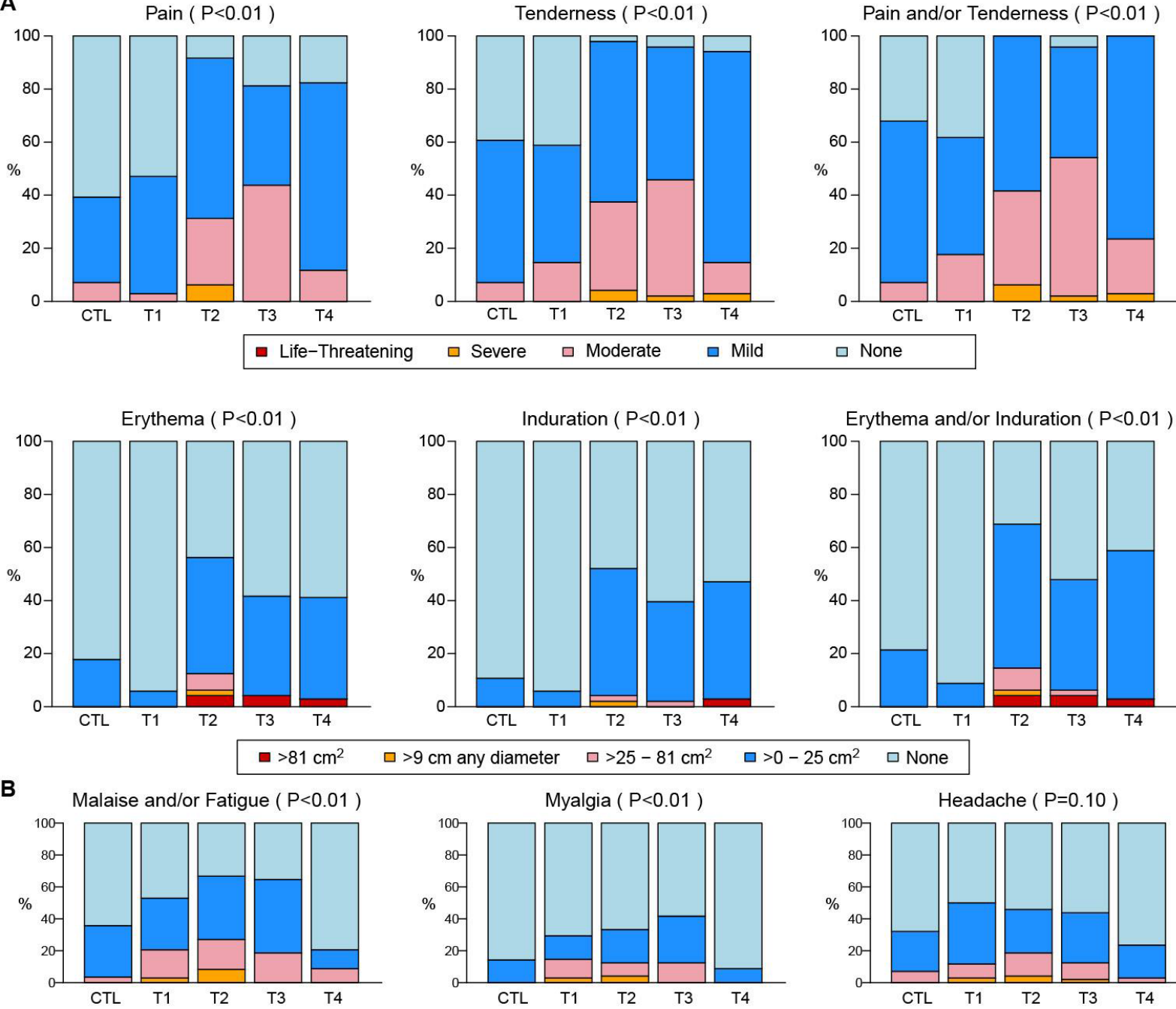

Erythema and/or Induration $(\mathrm{P}<0.01)$

$>9 \mathrm{~cm}$ any diameter $\quad \square>25-81 \mathrm{~cm}^{2}$
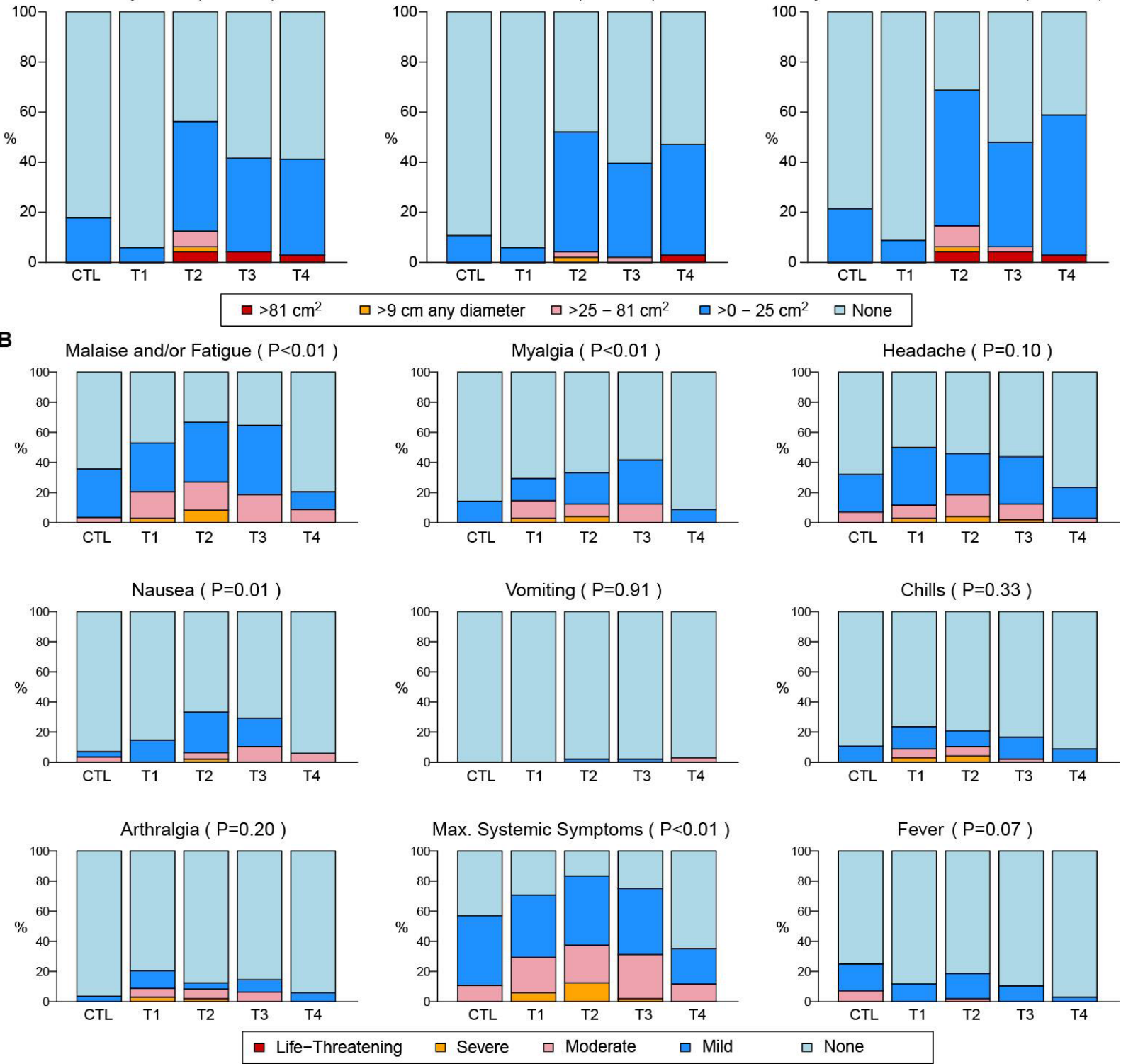

Figure 1: Safety assessment, showing maximum local reactogenicity A) and systemic reactogenicity, B) by treatment group. Subjects in control (C) groups 1-4 received group phosphate buffered saline. Subjects in treatment groups 1-3 (T1-T3) were Ad5 seronegative at baseline and received: T1- recombinant Ad35 (rAd35) prime and rAd5 boost; T2- three DNA priming injections boosted by rAd5; and T3- three DNA priming injections boosted by rAd35. Subjects in T4 were Ad5 seropositive at baseline and received three DNA priming injections boosted by rAd35. P values for comparisons of local and systemic reactogenicity were determined using the Kruskal-Wallis test. 
Citation: Fuchs JD, Bart PA, Frahm N, Morgan C, Gilbert PB, et al. (2015) Safety and Immunogenicity of a Recombinant Adenovirus Serotype 35-Vectored HIV-1 Vaccine in Adenovirus Serotype 5 Seronegative and Seropositive Individuals. J AIDS Clin Res 6: 461. doi:10.4172/21556113.1000461

\section{Clade B Env}

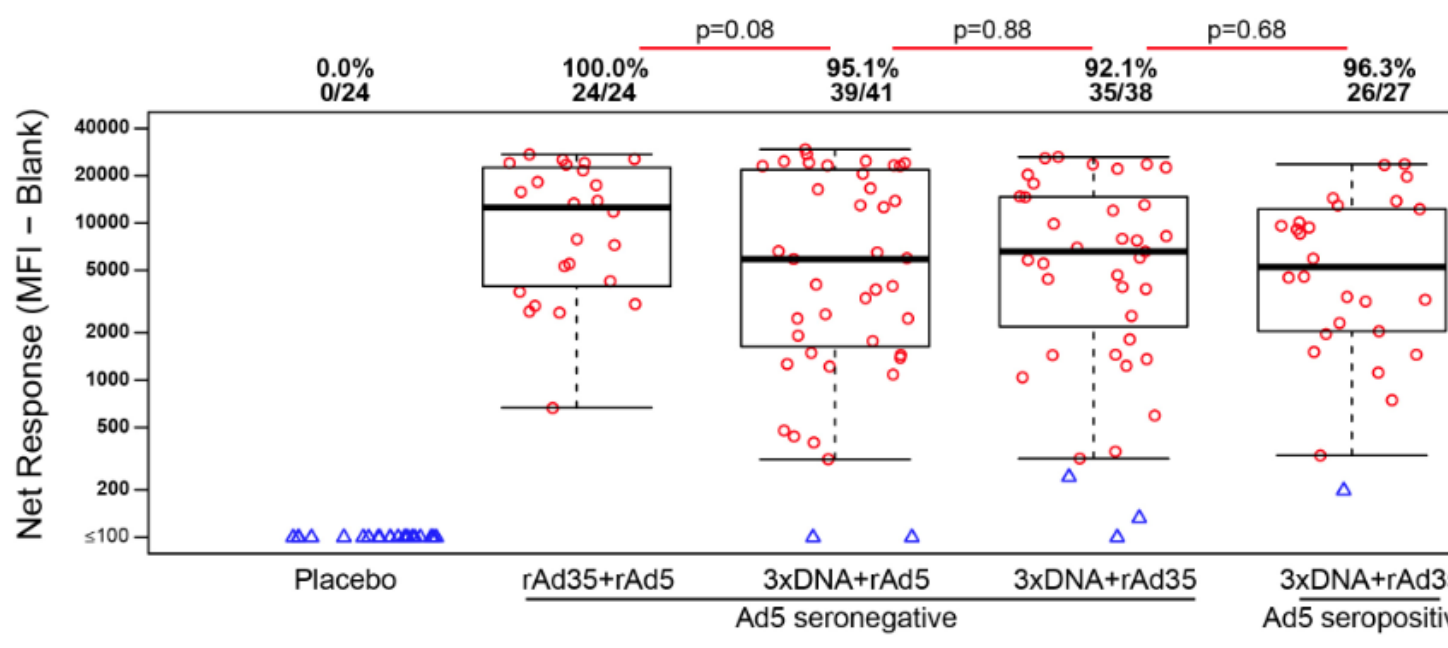

Clade C Env

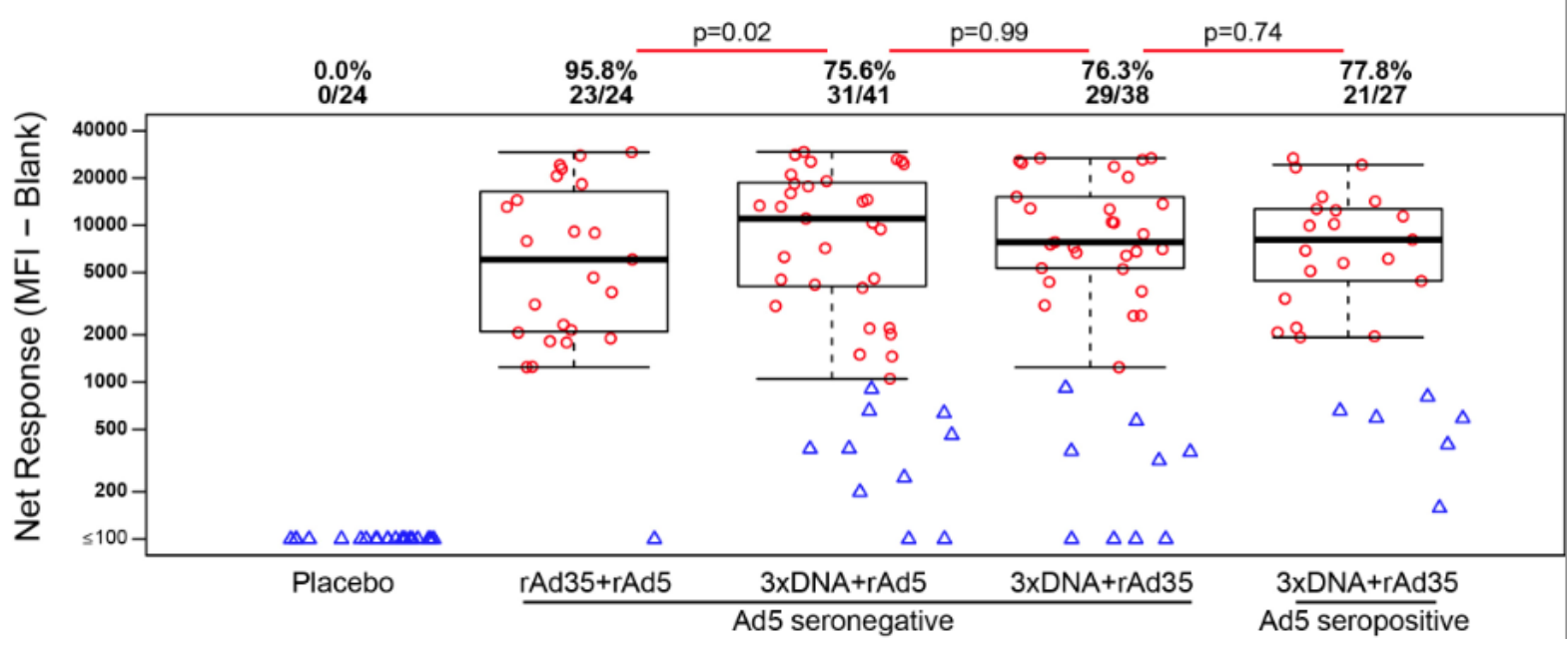

Figure 2: Binding antibody net responses to Clades A (OOMSA 4076 gp140), B (B.con.env03 140CF), and C (C.con.env03 140CF) isolates 4 weeks after the boost vaccination as measured by median fluorescence intensity (MFI)-Blank where 'Blank' is a sample specific background measure. Responders are shown in red circles and non-responders in blue triangles. Box plots display the distribution of positive responses for the vaccinees for each antigen. $P$-values are derived from Lachenbruch's test comparing rAd5 and rAd35 boosted groups in Ad5 seronegative individuals and the Ad35-boosted group in Ad5 seropositive individuals. 
Citation: Fuchs JD, Bart PA, Frahm N, Morgan C, Gilbert PB, et al. (2015) Safety and Immunogenicity of a Recombinant Adenovirus Serotype 35-Vectored HIV-1 Vaccine in Adenovirus Serotype 5 Seronegative and Seropositive Individuals. J AIDS Clin Res 6: 461. doi:10.4172/21556113.1000461

Page 6 of 10

efficacy trial [8], we explored whether the EnvA constructs tested in HVTN 077 elicited these responses. Among positive responders, all treatment groups elicited gp70V1/V2 responses using the V1/V2 scaffold tested in the RV144 study (Case A2, Figure 3A) and there were no significant differences by group in the magnitude or frequency of the responses. In addition, we looked at the matching clade A V1/ V2 sequence in the HVTN 077 vaccine regimen (clade A gp70V1/ V2). Although the frequency of response did not differ substantially by group, as seen in Figure 3B, the response magnitude for binding antibodies to the vaccine-matched clade A gp70V1/V2 was significantly higher among the DNA/rAd5 group compared to the rAd35/rAd5 group, $(\mathrm{p}=0.005)$.

\section{HIV-1-specific T-cell responses}

As seen in Figure 4, HIV-1-specific T cells producing IFN- $\gamma$ and/ or IL-2 in response to vaccine insert-matched peptides were detected readily in each of the treatment groups. With regard to the CD4+ T-cell responses, the highest post-boost response rates were seen among Ad5 seronegative individuals receiving DNA/rAd35 $(25 / 36,69.4 \%)$ and lowest among those receiving $\mathrm{rAd} 35 / \mathrm{rAd} 5$ (9/24, 37.5\%); responses did not differ significantly across groups. Vaccine-induced CD8+ T-cell responses were elicited most frequently among Ad5 seronegative individuals who received the DNA/rAd5 regimen $(32 / 42,76.2 \%)$ and least among Ad5 seropositives who received the DNA/rAd35 regimen (10/25, 40.0\%); responses did not differ significantly across groups. Overall, we found that among Ad5 seronegative subjects, $15 \%, 39 \%$, and $32 \%$ of rAd35/rAd5, DNA/rAd5, and DNA/rAd35 recipients, respectively, and $14 \%$ of Ad5 seropositive participants receiving the DNA/rAd5 regimen developed both CD4+ and CD8+ T-cell responses. The HIV-specific CD4+ or CD8+ T-cell response magnitudes for positive responders after boosting were similar across groups.

In addition, we assessed expression of TNF- $\alpha$ and Granzyme B $(\mathrm{GzB})$ in response to stimulation with insert-matched peptides. As shown in Figure 5, priming with DNA followed by either rAd35 or rAd5 led to the induction of significantly more polyfunctional CD4+ $\mathrm{T}$ cells than vaccination with $\mathrm{rAd} 35 / \mathrm{rAd} 5$ in Ad5 seronegative subjects $(p=0.0005$ for three and $p=0.007$ for four functions, respectively, comparing the combined DNA/rAd5 and DNA/rAd35 groups with $\mathrm{rAd} 35 / \mathrm{rAd} 5$ for Ad5 seronegative subjects). Interestingly, the patterns of combined expression of these functional markers did not

A

gp70 V1V2 (case A2)

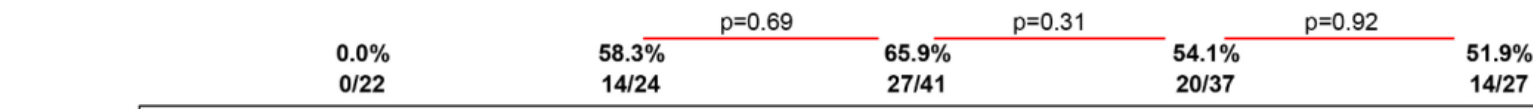
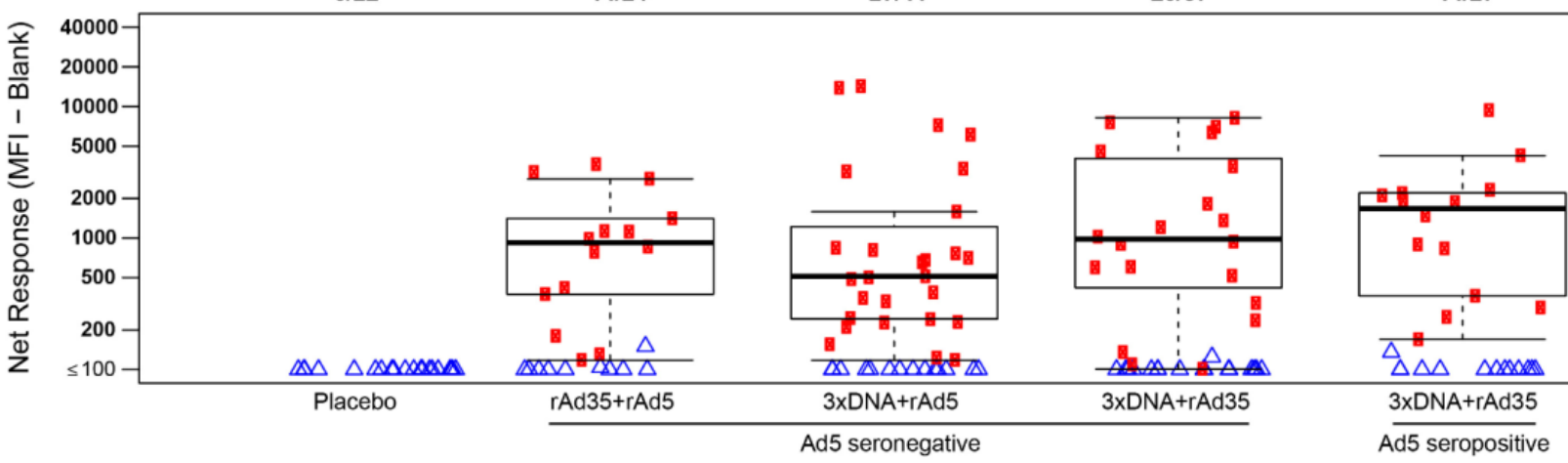

B

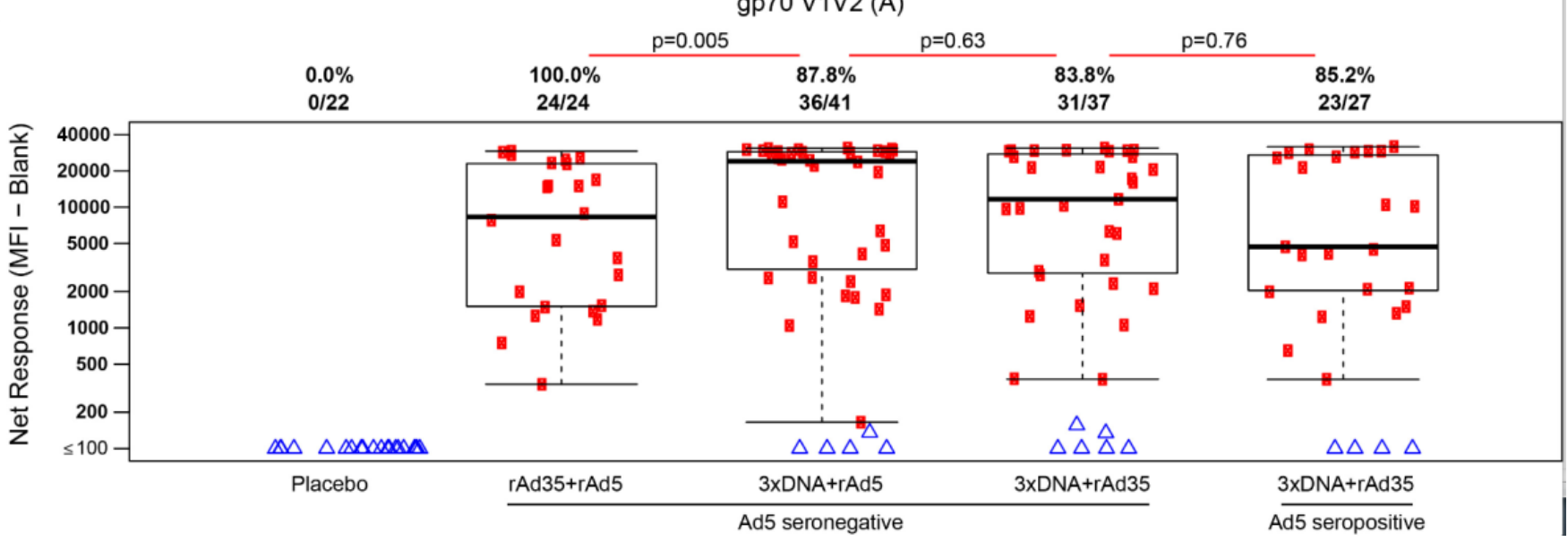

Figure 3: Binding antibody net responses to A) gp70 V1/V2 (Case A2) used in the RV144 trial and B) gp70 V1/V2 (A), the V1V2 antigen contained in the VRC A vaccine strain 4 weeks after the boost vaccination as measured by median fluorescence intensity (MFI)-Blank where 'Blank' is a sample specific background measure. Responders are shown in red circles and non-responders in blue triangles. Box plots display the distribution of positive responses for the vaccinees for each antigen. P-values are derived from Lachenbruch's test comparing rAd5 and rAd35 boosted groups in Ad5 seronegative individuals and the Ad35-boosted group in Ad5 seropositive individuals. 
Citation: Fuchs JD, Bart PA, Frahm N, Morgan C, Gilbert PB, et al. (2015) Safety and Immunogenicity of a Recombinant Adenovirus Serotype 35-Vectored HIV-1 Vaccine in Adenovirus Serotype 5 Seronegative and Seropositive Individuals. J AIDS Clin Res 6: 461. doi:10.4172/21556113.1000461

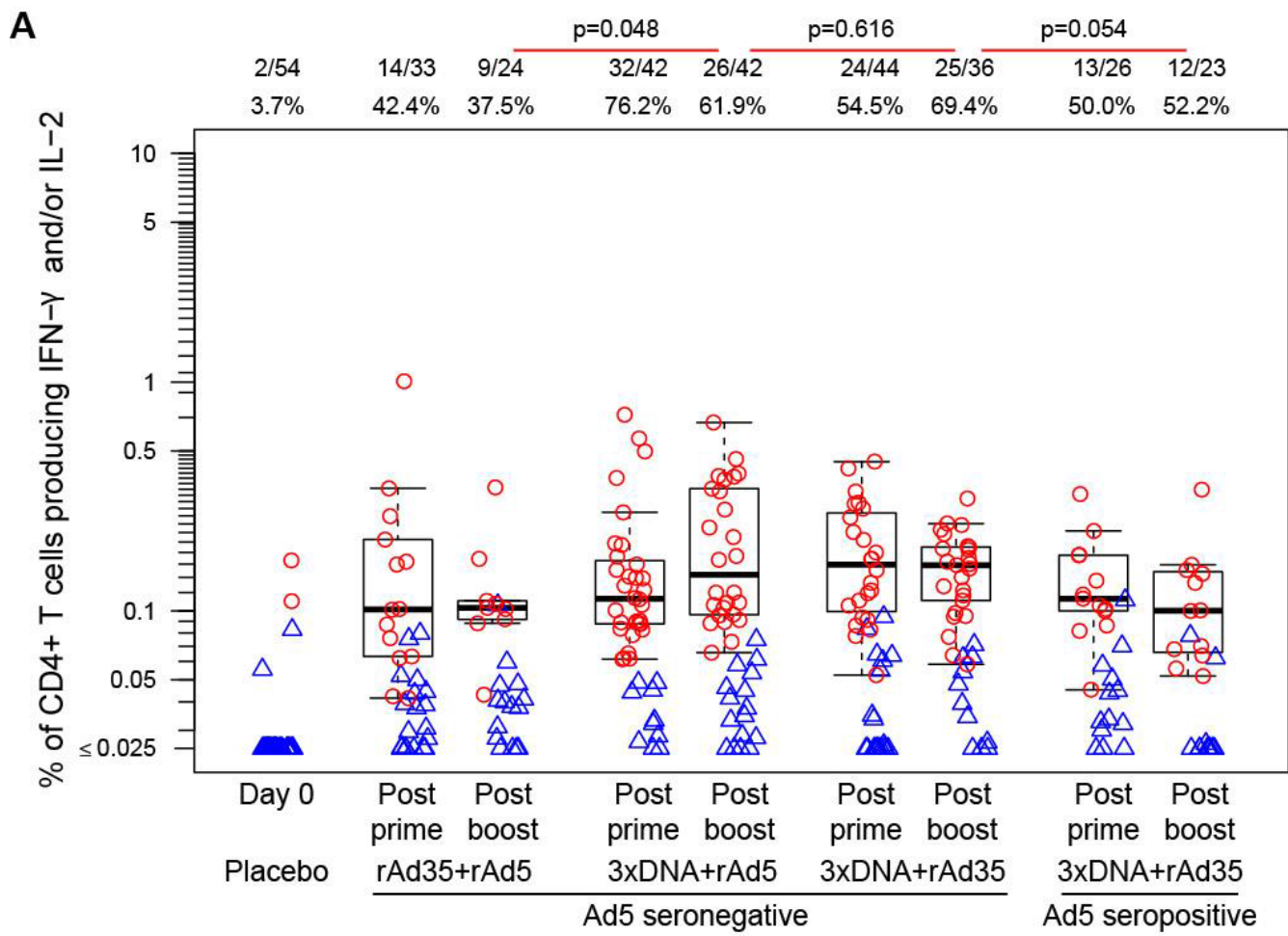

B

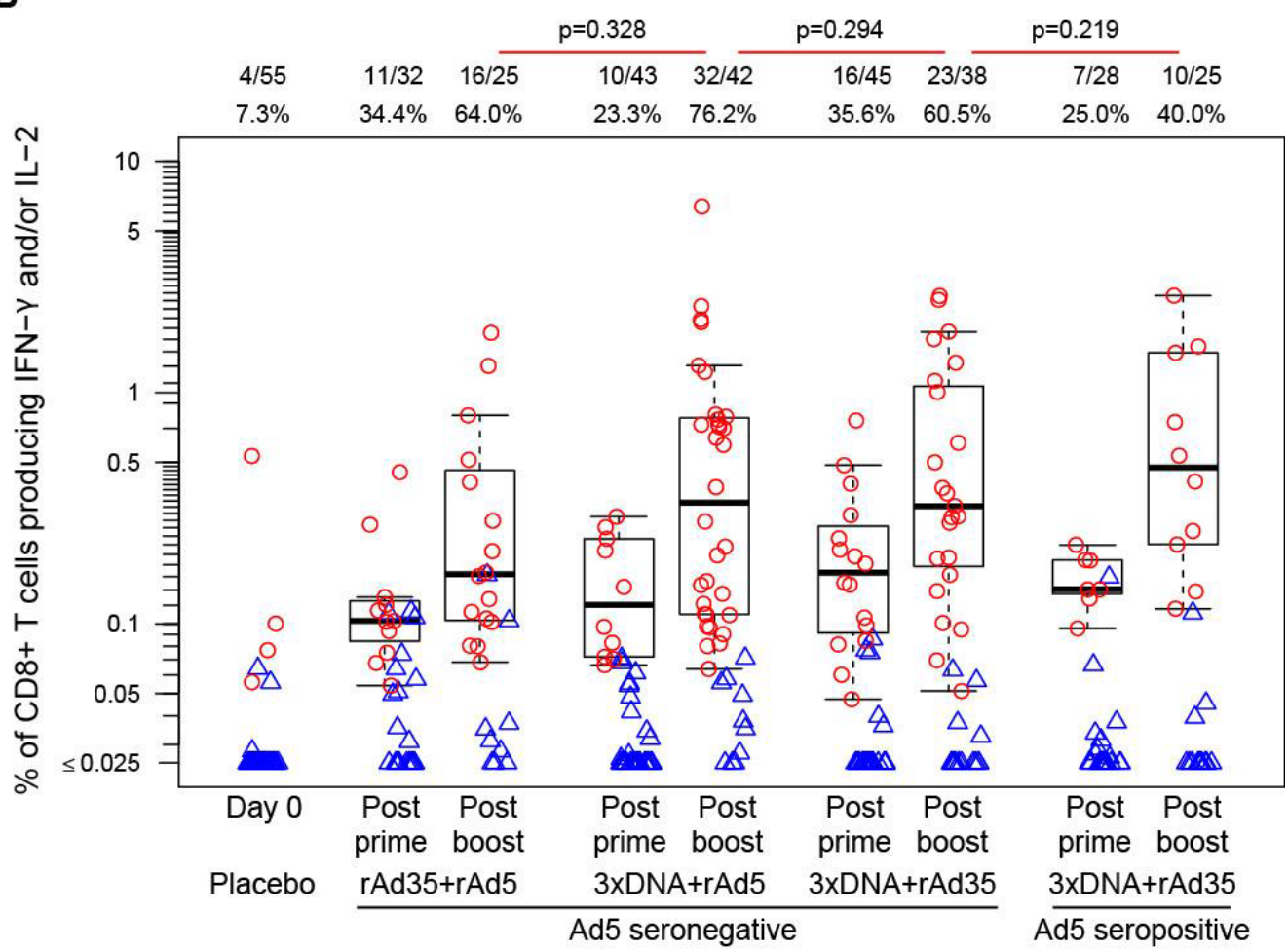

Figure 4. HIV-specific CD4+ and CD8+ T-cell responses: The percentage of CD4+ (panel A) and CD8+ (panel B) T cells producing $\gamma$-interferon (IFN- $\gamma$ ) and/or interleukin-2 (IL-2) in response to EnvA matched peptide pools 4 weeks after the priming immunization(s) and 4 weeks after the boost as measured by intracellular cytokine staining. Responders are shown in red circles and non-responders in blue triangles. Boxplots show the distribution of the magnitude of response in positive responders only. The box indicates the median and interquartile range (IQR); whiskers extend to the furthest point within 1.5 times the IQR from the upper or lower quartile. Numbers at the top of each panel show the number of responders / number with an assay result and the percent with positive response. P-values are derived from Lachenbruch's test comparing rAd5 and rAd35 boosted groups in Ad5 seronegative individuals and the Ad35-boosted group in Ad5 seropositive individuals. Data from samples with high background cytokine secretion was filtered, leading to differences in the number of samples with available data for CD4+ and CD8+ T cells. 


\section{CD4+}

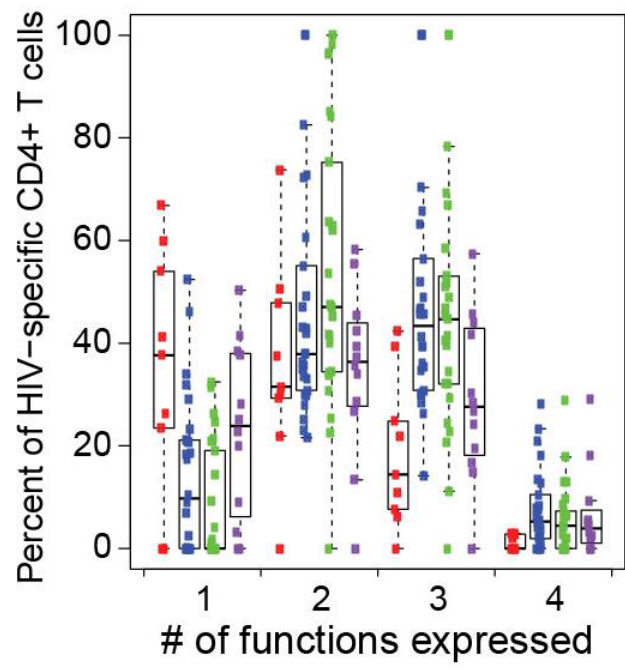

CD8+

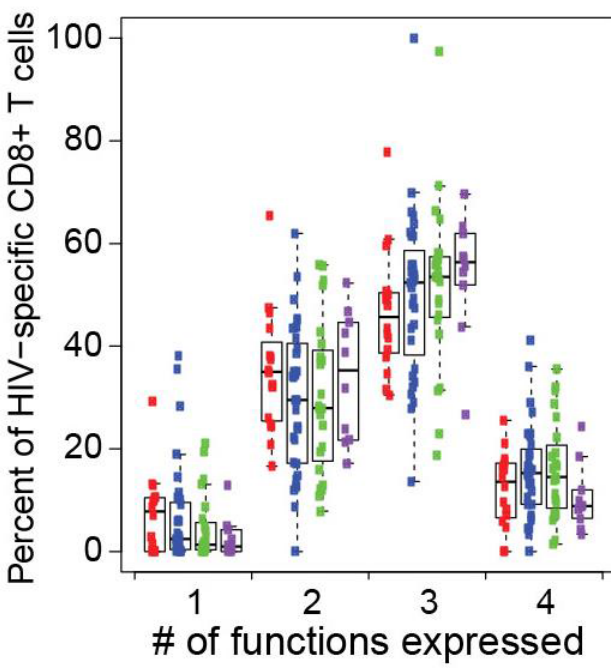

Figure 5. Vaccine-induced HIV-specific CD4+ and CD8+ T cells expressing multiple functional markers: The proportion of HIV-specific CD4+ (left) or CD8+ (right) $\mathrm{T}$ cells with one, two, three or four functions measured by expression of Granzyme B, IFN-g, IL-2, or TNFa is shown for positive responders from Figure 4 . Boxplots show the distribution of responses; the box indicates the median and IQR, whiskers extend to the furthest point within 1.5 times the IQR from the upper or lower quartile.

differ significantly between these groups after correction for multiple comparisons (Supplementary Figure 1). While around 90\% of HIVspecific CD8 $+\mathrm{T}$ cells were polyfunctional (Figure 5), only minor differences in expression patterns were observed among the different groups (Figure 5 and Supplementary Figure 1).

\section{Discussion}

In this clinical study of a prototype rAd 35 vectored HIV-1 vaccine, we found that it was well tolerated with a similar safety profile to that of rAd5-based experimental vaccines [12,34]. We sought to explore how the immunogenicity of the heterologous $\mathrm{rAd} 35 / \mathrm{rAd} 5$ regimen compared to a DNA/rAd5 regimen, and found that rAd35/rAd5 elicited similar frequency and magnitude of HIV-1-specific antibody responses, and slightly (although not statistically significant) lower $\mathrm{T}$ cell responses. In addition, we found that as a boost in DNA-primed subjects, there were no significant differences between rAd35 and rAd5 in the ability to induce EnvA-specific antibody and T-cell responses. We also observed that in the setting of pre-existing Ad5 nAbs, there was no significant reduction of HIV-1 specific antibody or cellular responses to an Ad35-based regimen when given once as a boost. This confirms findings from preclinical models that cross-reactive immune responses from these serologically distinct adenoviruses are unable to substantially dampen immunogenicity [23]. Finally, we found that each of the vaccine regimens tested in this trial was capable of inducing cross-clade binding antibodies as well as V1/V2-specific IgG antibodies, which were correlated with reduced risk of HIV infection in the RV144 vaccine efficacy trial [8].

The HVTN 505 Phase IIb efficacy trial showed that a DNA/rAd5 prime-boost regimen encoding EnvA, EnvB, EnvC, clade B Gag, Pol, and Nef was unable to prevent HIV acquisition or reduce viral load [7]. Of note, in HVTN 077, we found the proportion of vaccinees with V1/V2 IgG responses (52\% to $66 \%$ across the four groups) was similar to the rate observed for the partially efficacious ALVAC/gp120 vaccine regimen tested in the RV144 trial (64\%, 95\% CI 58\% to 70\%) [8] and higher than the $17.5 \%$ rate of response elicited by the HVTN 505 regimen
[7]. In addition, the $\mathrm{V} 1 / \mathrm{V} 2 \mathrm{IgG}$ responses were similar between groups 2 and 3 (DNA/rAd5 and DNA/Ad35 in Ad5 seronegative subjects), and group 4 (DNA/rAd35 in Ad5 seropositive subjects). This is in contrast to findings from the phase 2 study of the VRC multiclade DNA/rAd5 regimen [12], where $\mathrm{V} 1 / \mathrm{V} 2 \operatorname{IgG}$ responses were significantly lower in Ad5 seropositive vaccines compared to Ad5 seronegative individuals (G. Tomaras, personal communication). These findings suggest that presentation of the EnvA antigen alone may produce a more favorable antibody response to the V1/V2 region than presentation of multiple Env proteins with additional competing antigens. It is important to note that we do not know if the V1/V2 IgG correlate of risk translates into a correlate of protection, and, if so, whether it is a mechanistic or nonmechanistic correlate [35] or whether IgG V1/V2 responses will be a correlate of HIV-1 risk or protection for vaccines in other populations that differ from the community-based sample evaluated in Thailand [6]. However, studies in non-human primate models suggest that envelope binding antibodies, V2-specific antibodies, and the avidity to which anti-Env antibodies bind to native trimer [18,36-38] can correlate with protection. Therefore, future HIV-1 vaccines designed to elicit Env binding responses and V2-specific IgG antibodies, should consider these observations from preclinical studies and recent clinical trials.

Whether multi-dose DNA priming for an adenovector boost confers an immunologic advantage over heterologous adenovector regimens is relevant given the desire for less complex vaccine regimens with fewer required immunizations. Compared to $\operatorname{rAd} 35 / \mathrm{rAd} 5$, we found that the DNA/rAd5 regimen elicited higher magnitude binding antibody responses to some of the antigens tested (e.g., the EnvA clade-matched V1/V2 expressed by the vaccine and EnvC) but not to others (e.g, the Clade A Env). And while there was no overall difference in the HIVspecific CD4+ or CD8+ T-cell responses elicited by these regimens, DNA priming may generate more polyfunctional responses than the heterologous adenovector prime-boost regimen, a desirable feature of vaccines designed to elicit cellular immunity $[39,40]$. Several studies in the field will provide further insights into the relative immunogenicity of prime-boost regimens combining different adenovectors such as 
Citation: Fuchs JD, Bart PA, Frahm N, Morgan C, Gilbert PB, et al. (2015) Safety and Immunogenicity of a Recombinant Adenovirus Serotype 35-Vectored HIV-1 Vaccine in Adenovirus Serotype 5 Seronegative and Seropositive Individuals. J AIDS Clin Res 6: 461. doi:10.4172/21556113.1000461

Page 9 of 10

Ad35 and Ad26 encoding an EnvA antigen (IAVI B003-IPCAVD004HVTN091, NCT01215149) and DNA administered by electroporation with a multi-antigen rAd35 construct (IAVIB004; NCT01496989). Furthermore, to optimize immunogenicity, trials should carefully consider the administration interval between adenovector prime and boost. Our study delivered rAd35 and rAd5 6 months apart, eliciting higher magnitude HIV-specific T-cell responses compared to rAd35/ rAd5 given only 3 months apart in VRC 012 (NCT00479999) [22].

\section{Conclusion}

In this phase $1 \mathrm{~b}$ study, we have demonstrated that $\mathrm{rAd} 35$ is well tolerated and immunogenic, and as a boost, is as potent as rAd5 in DNA primed individuals. In addition, the humoral and cellular responses elicited by rAd35 boosting are better preserved in the setting of preexisting Ad5 seropositivity than responses to rAd5 boosting, suggesting that rAd35 is a reasonable choice for an alternative adenoviral vaccine vector to diminish the impact of antivector immunity. Therefore, as additional safety data emerge from studies exploring alternative adenoviral vectors in different global contexts, rAd35 should be considered for use as a vaccine delivery vector, particularly as effective antigen designs become available. This is particularly relevant when CD8+ T cell-mediated immunity is desirable in addition to antibodybased immunity, in subjects already primed with the antigens expressed by the rAd35 vector, and in settings with a high prevalence of preexisting immunity to Ad5, such as in sub-Saharan Africa.

\section{Acknowledgments}

The authors would like to thank Drs. Denny Kim, Marcel Curlin, and ChuenYen Lau for their involvement in early study implementation. Dr. Gary Nabel for access to reagents to probe V1V2 antibody responses, and Stephen Voght for his assistance with preparation of the tables and figures herein. They would also like to acknowledge the contributions of the HVTN 077 study staff, the sites' community advisory boards, and the clinical trial participants.

\section{Author contributions}

Designed the study: JF, PAB, PG, NF, SD, TW, BG*, MJM. Oversaw study conduct and managed participants at study sites: JF, PAB, LB, BK, MS, KM, PG NR, SK, MK, MJM. Medical monitoring and study oversight: JF, PAB, ES*, CM, $\mathrm{BG}^{*}$. Oversaw performing immunogenicity assays and result interpretation: JF, NF $\mathrm{SD}, \mathrm{SR}, \mathrm{GT}, \mathrm{JM}, \mathrm{BG}^{*}$. Analyzed the data: JF, NK, PG, NF, GT, BG*, MJM. Wrote the manuscript: JF, PG, NF, GT, BG, MJM.

*This paper was written by authors in their capacity as NIH employees, but the views expressed in this paper do not represent those of the $\mathrm{NIH}$.

\section{Financial Support}

HVTN 077 was conducted by the HIV Vaccine Trials Network (HVTN) and supported by the National Institutes of Allergy and Infectious Diseases (NIAID-NIH). This work was also supported by the following grants: UM1 AI068614 (HVTN Core FHCRC), UM1 AI068635 (SCHARP), UM1 AI068618 (HVTN Laboratory Program), UM1 AI069452 (UAB), UM1AI069418 (Emory), UM1AI069511 (Rochester), UM1 Al069412 (BWH and Fenway), UM1 Al069470 (NY Blood Center -Bronx, NY Blood Center -Union Square and Columbia University), UM1 AI069439 (Vanderbilt), UM1 Al069496 (SFDPH), and UM1 AI069481 (Seattle)

Clinical trials Registration. NCT00801697

\section{References}

1. Fact Sheet (2014) Global Statistics. Joint United Nations Programme on HIV/ AIDS (UNAIDS).

2. Flynn NM, Forthal DN, Harro CD, Judson FN, Mayer KH, et al. (2005) Placebocontrolled phase 3 trial of a recombinant glycoprotein 120 vaccine to prevent HIV-1 infection. J Infect Dis 191: 654-665.

3. Pitisuttithum P, Gilbert P, Gurwith M, Heyward W, Martin M, et al. (2006) Randomized, double-blind, placebo-controlled efficacy trial of a bivalent recombinant glycoprotein $120 \mathrm{HIV}-1$ vaccine among injection drug users in Bangkok, Thailand. J Infect Dis 194: 1661-1671.

4. Buchbinder SP, Mehrotra DV, Duerr A, Fitzgerald DW, Mogg R, et al. (2008)
Efficacy assessment of a cell-mediated immunity HIV-1 vaccine (the Step Study): a double-blind, randomised, placebo-controlled, test-of-concept trial. Lancet 372: 1881-1893.

5. Gray GE, Allen M, Moodie Z, Churchyard G, Bekker LG, et al. (2011) Safety and efficacy of the HVTN 503/Phambili study of a clade-B-based HIV-1 vaccine in South Africa: a double-blind, randomised, placebo-controlled test-of-concept phase 2b study. Lancet Infect Dis 11: 507-515

6. Rerks-Ngarm S, Pitisuttithum P, Nitayaphan S, Kaewkungwal J, Chiu J, et al. (2009) Vaccination with ALVAC and AIDSVAX to prevent HIV-1 infection in Thailand. N Engl J Med 361: 2209-2220.

7. Hammer SM, Sobieszczyk ME, Janes H, Karuna ST, Mulligan MJ, et al. (2013) Efficacy trial of a DNA/rAd5 HIV-1 preventive vaccine. N Engl J Med 369: 20832092.

8. Haynes BF, Gilbert PB, McElrath MJ, Zolla-Pazner S, Tomaras GD, et al. (2012) Immune-correlates analysis of an HIV-1 vaccine efficacy trial. N Engl J Med 366: $1275-1286$.

9. De Rosa SD, McElrath MJ (2008) T cell responses generated by HIV vaccines in clinical trials. Curr Opin HIV AIDS 3: 375-379.

10. Duerr A, Huang Y, Buchbinder S, Coombs RW, Sanchez J, et al. (2012) Extended follow-up confirms early vaccine-enhanced risk of HIV acquisition and demonstrates waning effect over time among participants in a randomized trial of recombinant adenovirus HIV vaccine (Step study). J Infect Dis 206: 258266.

11. Koup RA, Roederer M, Lamoreaux L, Fischer J, Novik L, et al. (2010) Priming immunization with DNA augments immunogenicity of recombinant adenoviral vectors for both HIV-1 specific antibody and T-cell responses. PLoS One 5: e9015.

12. Churchyard GJ, Morgan C, Adams E, Hural J, Graham BS, et al. (2011) A phase IIA randomized clinical trial of a multiclade HIV-1 DNA prime followed by a multiclade rAd5 HIV-1 vaccine boost in healthy adults (HVTN204). PLoS One 6: e21225.

13. Kostense S, Koudstaal W, Sprangers M, Weverling GJ, Penders G, et al. (2004) Adenovirus types 5 and 35 seroprevalence in AIDS risk groups supports type 35 as a vaccine vector. AIDS 18: 1213-1216.

14. Nwanegbo E, Vardas E, Gao W, Whittle H, Sun H, et al. (2004) Prevalence of neutralizing antibodies to adenoviral serotypes 5 and 35 in the adult populations of The Gambia, South Africa, and the United States. Clin Diagn Lab Immunol 11: $351-357$

15. Vogels R, Zuijdgeest D, van Rijnsoever R, Hartkoorn E, Damen I, et al. (2003) Replication-deficient human adenovirus type 35 vectors for gene transfer and vaccination: efficient human cell infection and bypass of preexisting adenovirus immunity. J Virol 77: 8263-8271.

16. Barouch DH, Kik SV, Weverling GJ, Dilan R, King SL, et al. (2011) International seroepidemiology of adenovirus serotypes $5,26,35$, and 48 in pediatric and adult populations. Vaccine 29: 5203-5209.

17. Gaggar A, Shayakhmetov DM, Lieber A (2003) CD46 is a cellular receptor for group B adenoviruses. Nat Med 9: 1408-1412.

18. Barouch DH, Liu J, Li H, Maxfield LF, Abbink P, et al. (2012) Vaccine protection against acquisition of neutralization-resistant SIV challenges in rhesus monkeys. Nature 482: 89-93.

19. Keefer MC, Gilmour J, Hayes P, Gill D, Kopycinski J, et al. (2012) A phase I double blind, placebo-controlled, randomized study of a multigenic HIV-1 adenovirus subtype 35 vector vaccine in healthy uninfected adults. PLoS One 7: e41936.

20. Hoft DF, BlazevicA, Stanley J, Landry B, Sizemore D, et al. (2012) A recombinan adenovirus expressing immunodominant TB antigens can significantly enhance BCG-induced human immunity. Vaccine 30: 2098-2108.

21. Barnes E, Folgori A, Capone S, Swadling L, Aston S, et al. (2012) Nove adenovirus-based vaccines induce broad and sustained $T$ cell responses to HCV in man. Sci Transl Med 4: 115ra1.

22. Graham BS, Ledgerwood JE, Novik L, Enama ME, Hendel CS, et al. (2010) Safety and immunogenicty of a rAd35-EnvA prototype HIV-1 vaccine in combination with rAd5-EnvA in healthy adults (VRC012) [abstract P14.11LB] In: Program and abstracts of AIDS Vaccine 2010 (Atlanta, GA).

23. Barouch DH, Pau MG, Custers JH, Koudstaal W, Kostense S, et al. (2004) 
Citation: Fuchs JD, Bart PA, Frahm N, Morgan C, Gilbert PB, et al. (2015) Safety and Immunogenicity of a Recombinant Adenovirus Serotype 35-Vectored HIV-1 Vaccine in Adenovirus Serotype 5 Seronegative and Seropositive Individuals. J AIDS Clin Res 6: 461. doi:10.4172/21556113.1000461

Page 10 of 10

Immunogenicity of recombinant adenovirus serotype 35 vaccine in the presence of pre-existing anti-Ad5 immunity. J Immunol 172: 6290-6297.

24. Aste-Amézaga M, Bett AJ, Wang F, Casimiro DR, Antonello JM, et al. (2004) Quantitative adenovirus neutralization assays based on the secreted alkaline phosphatase reporter gene: application in epidemiologic studies and in the design of adenovector vaccines. Hum Gene Ther 15: 293-304.

25. Sprangers MC, Lakhai W, Koudstaal W, Verhoeven M, Koel BF, et al. (2003) Quantifying adenovirus-neutralizing antibodies by luciferase transgene detection: addressing preexisting immunity to vaccine and gene therapy vectors. J Clin Microbiol 41: 5046-5052.

26. Tomaras GD, Yates NL, Liu P, Qin L, Fouda GG, et al. (2008) Initial B-cell responses to transmitted human immunodeficiency virus type 1: virion-binding immunoglobulin M (IgM) and IgG antibodies followed by plasma anti-gp41 antibodies with ineffective control of initial viremia. J Virol 82: 12449-12463.

27. Liao HX, Bonsignori M, Alam SM, McLellan JS, Tomaras GD, et al. (2013) Vaccine induction of antibodies against a structurally heterogeneous site of immune pressure within HIV-1 envelope protein variable regions 1 and 2 . Immunity 38: 176-186.

28. Pinter A, Honnen WJ, Kayman SC, Trochev O, Wu Z (1998) Potent neutralization of primary HIV-1 isolates by antibodies directed against epitopes present in the V1/V2 domain of HIV-1 gp120. Vaccine 16: 1803-1811.

29. Bull M, Lee D, Stucky J, Chiu YL, Rubin A, et al. (2007) Defining blood processing parameters for optimal detection of cryopreserved antigen-specific responses for HIV vaccine trials. J Immunol Methods 322: 57-69.

30. Horton H, Thomas EP, Stucky JA, Frank I, Moodie Z, et al. (2007) Optimization and validation of an 8-color intracellular cytokine staining (ICS) assay to quantify antigen-specific $\mathrm{T}$ cells induced by vaccination. J Immunol Methods 323: 39-54.

31. McElrath MJ, De Rosa SC, Moodie Z, Dubey S, Kierstead L, et al. (2008) HIV1 vaccine-induced immunity in the test-of-concept Step Study: a case-cohort analysis. Lancet 372: 1894-1905.

32. Frahm N, DeCamp AC, Friedrich DP, Carter DK, Defawe OD, et al. (2012) Human adenovirus-specific T cells modulate HIV-specific $T$ cell responses to an Ad5-vectored HIV-1 vaccine. J Clin Invest 122: 359-367.

33. Lachenbruch PA (2001) Comparisons of two-part models with competitors. Stat Med 20: 1215-1234

34. Koblin BA, Casapia M, Morgan C, Qin L, Wang ZM, et al. (2011) Safety and immunogenicity of an HIV adenoviral vector boost after DNA plasmid vaccine prime by route of administration: a randomized clinical trial. PLoS One 6: e24517.

35. Plotkin SA, Gilbert PB (2012) Nomenclature for immune correlates of protection after vaccination. Clin Infect Dis 54: 1615-1617.

36. Strbo N, Vaccari M, Pahwa S, Kolber MA, Doster MN, et al. (2013) Cutting edge: novel vaccination modality provides significant protection against mucosal infection by highly pathogenic simian immunodeficiency virus. J Immunol 190 : 2495-2499.

37. Zhao J, Lai L, Amara RR, Montefiori DC, Villinger F, et al. (2009) Preclinica studies of human immunodeficiency virus/AIDS vaccines: inverse correlation between avidity of anti-Env antibodies and peak postchallenge viremia. J Virol 83: 4102-4111.

38. Lai L, Kwa S, Kozlowski PA, Montefiori DC, Ferrari G, et al. (2011) Prevention of infection by a granulocyte-macrophage colony-stimulating factor co-expressing DNA/modified vaccinia Ankara simian immunodeficiency virus vaccine. J Infect Dis $204: 164-173$.

39. Precopio ML, Betts MR, Parrino J, Price DA, Gostick E, et al. (2007) Immunization with vaccinia virus induces polyfunctional and phenotypically distinctive CD8(+) T cell responses. J Exp Med 204: 1405-1416.

40. Gaucher D, Therrien R, Kettaf N, Angermann BR, Boucher G, et al. (2008) Yellow fever vaccine induces integrated multilineage and polyfunctional immune responses. J Exp Med 205: 3119-3131. 